\title{
P-0862. Does body weight perception and/or actual body weight influence physical activity and quality of life of adults?
}

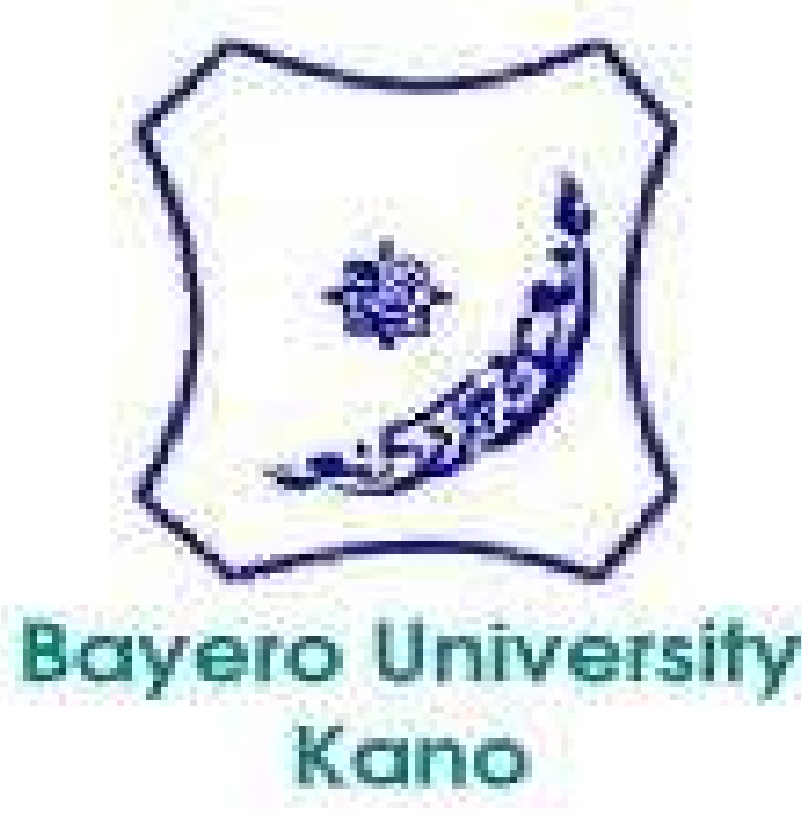

KWAZULU-NATAL

\section{F.A. Maruf, ${ }^{1,3}$ Ph.D., O.H Ugwu, ${ }_{1}$ BMR (PT), B. Kaka,2,3 Ph.D., S.S Maharaj Ph.D.}

1, Department of Medical Rehabilitation, Faculty of Health Sciences and Technology, Nnamdi Azikiwe University, Nnewi Campus, Nnewi, Anambra State, Nigeria.

2, Divisions of Physiotherapy, School of Health Sciences, College of Health Sciences, University of KwaZulu-Natal, Westville, Durban, South Africa

3, Department of Physiotherapy, Faculty of Allied Health Sciences, College of Health Sciences, Bayero University Kano. Email; bkaka.pth@buk.edu.ng

\section{Introduction}

Body size perception is the belief, opinion or insight into one's body size based on physical appearance (Grogan, 2016). It is a complicated construct that is composed of several components such as mental and emotional components, perceptual components and behavioral components(Nayir, Uskun, Yürekli, Devran, \& Çelik, 2016). It depends on psychological factors and socio-cultural influences such as family, peers, and ethnicity(Gualdi-Russo et al., 2012). Actual body size, however, is one of several factors that shape how one perceives one's weight(Chang \& Christakis, 2003). Wrongful perception of small body size may act as a barrier to physical activity (PA) behavior and may also affect the quality of life (QoL). The purpose of this study is to determine BMI, perceived body size/weight and their influences on PA level and QoL among adult residents in Nnewi.

\section{Methods}

Three hundred and fifty-seven residents of Nnewi participated in this cross-sectional survey. They were recruited consecutively using nonprobability sampling technique. The participants comprised different groups of residents such as market people, artisans, motor park workers, teachers, civil servants, and students. The participants were included if they had lived in Nnewi for at least one year, were within the age of 18-65 and could speak English or Igbo language. All the participants gave their consent to be part of the study. Socio-demographic information was collected using a biodata form. BMI, perceived body size/weight, PA level and QoL were measured using standardized procedures. Data were analyzed using descriptive and inferential statistics

\section{Results}

Most of the participants perceived their body size $(64.4 \%)$ as normal. However, their actual body size (ABS) was observed to be predominantly normal weight (40.3\%) and overweight (39.7\%). BMI significantly influenced PA $(p<0.01)$ and Mental component score of QoL $(p<0.01)$. However, only PA level was significantly influenced by perceived body size $(p<0.01)$, perceived body weight $(p<0.01)$, perceived ideal body weight $(p<0.01)$.

\section{Discussion}

The aim of this study was to determine BMI, perceived body size/weight and their influences on PA level and QoL among adult residents in Nnewi. The findings from this study showed that majority of participants had normal weight and overweight categories of BMI. However, they most frequently reported normal weight as their perceived body size, perceived body weight, and ideal body size. Also, they perceived their level of fatness to be about right. PA level and MC score of QoL differed significantly across BMI categories. These findings were true for only males when the analyses were carried out by gender. However, only PA level differed across perceived body size, perceived body weight, perceived ideal body size and perceived level of fatness categories. When these analyses were carried out by gender, perceived body weight did not influence PA levels in either males or females, but perceived body weight influence PA level in males and perceived ideal body image did in females.

\section{Conclusion}

PA level varies among adult individuals in this study regardless perceived body size/weight or BMI category. Although BMI category influences the MC score of QoL, the participants' perception of their body size/weight did not influence QoL. Perceived body size/weight should be taken into consideration in any health education programme to increase Physical activity behavior. 